\title{
Employing Blockchain Technology to Understand Personalized Medicine
}

\author{
Jack Kushner* \\ Head Prof Med, MD, Annapolis, Maryland
}

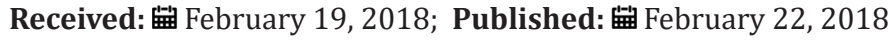

*Corresponding author: Jack Kushner, Head Prof Med, MD, Annapolis, Maryland, Tel: 4107573754; Email: jkaoportal@comcast.net

\begin{abstract}
Blockchain technology is yet another method in helping in the understanding and employment of personalized medicine. This technology can complement the use of mathematical models for the amalgamation of complex data from genetics, sequencing, mutations, cellular reactions, interactomes, medications, medication dosing and scheduling, and drug reactions. Additional uses of blockchain technology are described.
\end{abstract}

Keywords: Blockchain Technology; Personalized Medicine; Computational Mathematics; Genetics; Drug Delivery Systems; Interactomes

\section{Introduction}

In order to interpret the vast amount of information available on sequencing the genetic composition of patients, the data on analyzing diseases, genetic mutations, cellular reactions with genes, interactomes, medications, drug reactions and toxicities, doses of drugs and therapeutic schedules, and epidemiology problems, it is necessary to have secure methods with which to work. Reference is made to an earlier discussion on the use of computational mathematics and mathematical models for understanding personalized medicine. Kushner [1]. This article seeks to explain the benefits of yet another method available to decipher the vast amount of data that is being generated. Recently there has been a tremendous amount of discussion and publicity about crypto currency and blockchain technology among public and financial institutions. Most of this has been centered on financial transfers using Bit coin and other crypto currency avenues.

And while this has been in the limelight, there has been a quieter and more subtle revolution with the application of blockchain technology in the medical world. Blockchain creates a chain of transaction blocks to store information. One reason why there is such an attraction to blockchain technology is that this technique enables data exchange to occur in a secure and irrevocable world. Blockchain technology can deal with medical claim adjudication and billing management, as well as controlling the supply of drugs and aiding in the avoidance of counterfeit medicines. Perhaps one of the most obvious applications of this technology would be in the field of management of health care paper records and the electronic health care record. This technology permits the sharing of patient information by increasing the speed and availability of research data.

In order to make good decisions in medical research and personalized medicine, the employment of blockchain technology can aid in determining treatment protocols and medical choices. In fact, clinical trials with various medications will be suspect if there is missing data, endpoint switching, or data dredging. Polacheck [2]. Now health care professionals are interested in blockchain technology because the extended functionality of the blockchain and the emerging application program interface (API) services that enable several collaborative service processes. A transaction block is replicated across a collection of computers connected as a peer-to-peer network that constitutes a blockchain. Each of these computers is referred to as a node. Advanced cryptography allows for the nodes to interact anonymously and securely on the network. Culver [3]. Boderson C et al. [4] notes that another definition would be: "A distributed tamperproof database that secures all records that are added to it, wherever they exist. Each record contains a timestamp and secure links to the previous record" Boderson C et al. [4].

A block of one or more new transactions is collected into the transaction data. A series of hashes connect via the header, and with the header referring to the previous hashed copy thus forming the ability to chain together transactions. Copies of each transaction are hashed, and the hashes are paired, hashed, paired again, and 
hashed again until a single hash remains Stagnaro [5]. Broder et al. [6] writes "Blockchains are cryptographic protocols that allow a network of computers (nodes) collectively to maintain shared ledger of information without the need for complete trust between the nodes. Each blockchain database is a time-sequenced chain of events that have been authenticated using a consensus mechanism specified by the protocol. The mechanism guarantees that, as long as the majority of the network validates the blocks posted to the ledger as per the stated governance rules, information stored on the blockchain can be trusted as reliable. The effect of the distributed consensus mechanisms often means that all of the nodes of the network hold all the information stored on the blockchain" Broder et al. [6].

Blockchain technology can be used in five data-driven areas:
a. Longitudinal health care records
b. Automated health care claims adjudication
c. Interoperability
d. Online patient access
e. Supply chain management.

This technology has an extended functionality, which enables collaborative and operational services that deal with data-sharing. Das [5,7]. Longitudinal health care records use blockchain to link various healthcare provider organizations. For example, Weiss gives an example in which a patient has an acute episode and is attended by an emergency medical technician (EMT), who swipes the patient's interactive wristband containing his healthcare blockchain ID number. This information is broadcast to the patient's primary care doctor and to the hospital. Using a blockchain encryption security key to obtain messaging, they can access the patient's s updated blockchain health care account Weiss M [8].

A second use of blockchain in healthcare concentrates on automated health claim adjudication which ensures correct completion of the claims and supports compliance audits using business rules. This process allows payers to send remittances to providers and allows patients to process payments using their bank or health savings accounts Miliard M [9]. The third use of blockchain for this discussion involves the gathering of massive amounts of patient data. This technique supports HIPAA'S Protected Health Information federal laws and regulations. This use of blockchain for personalized medicine complements the earlier article on the employment of computational mathematics and mathematical models to deal with large amounts of important clinical and research medical data Kushner et al. [1].

The fourth use of blockchain technology in healthcare allows for a patient's access to his healthcare records. The patient is provided with a security key that matches his provider's key. Eckblaw has proposed a variation of the technique, which is used by a company named "MedRec". This company uses Ethereum, a clinical and research blockchain, for their medical records, and believes that this provides improved data quality for medical research. Eckblaw A et al. [10]. And finally, blockchain technology can be used for supply chain management by providing real time contract tracking, execution, and the ability to determine if a satisfactory completion of the contracts has been obtained. Thus information will be available concerning the ingredients, quality, and source of drugs being purchased. This could aid in the fight against counterfeit drugs being sold worldwide.

The quest for managing big data is not limited to health care, but is a problem for many organizations and corporations. Jennifer Bresnick has proposed the creation of an ecosystem that stresses accuracy, timeliness, and shared-decision making Bresnick J [11]. Personalized medicine would benefit by the use of blockchain technology in integrating all of the patient's health data by using tools such as the American College of Surgeons' National Surgical Quality Improvement Program (ACS NSQUIP) Surgical Risk Calculator. If all of the patient's data were already on his electronic health care record, then the cost of manually including this information on databases such as the National Trauma Data Bank and the National Cancer Database could be reduced.

In addition, all genetic data and phenotypic data from online sources and from wearable data could be integrated into the electronic health care record Peters AW et al. [12]. Blockchain technology can help solve some of the problems experienced with the present health IT systems since interoperability is critical to the Precision Medicine Initiative (PMI). Linn and Koo have suggested that all medical data could be stored in a data lake which is scalable Linn L [13]. These data lakes could be used for determining the best treatment based on the genetic information obtained from text mining, text analytics, and machine learning. All of this information would be encrypted and digitally signed to ensure privacy. Blockchain works with standard algorithms and protocols for cryptography and data encryption. In summary, blockchain technology may be used in conjunction with computational mathematics and mathematical models to determine whether or not the disease originates from a known specific genetic defect. This technology can tract whether or not the data supports the diagnosis and the treatment recommended. And finally, the massive amount of data should reveal the effectiveness of any medications proposed.

\section{References}

1. Kushner J, Buchanan JL (2017) Employing Mathematical Models to Understand Personalized Medicine. EC Microbiology 12.4(2017): 196201.

2. Polachek N, Maven W, Blockchain in Healthcare, USA.

3. Culver K (2016) Blockchain Technologies: A Whitepaper Discussing How the Claims Process can be improved. p. 1-10.

4. Boderson C, Kalis B, Leong C, Mitchell E, Pupo E, et al. (2016) Blockchain: Securing a New Health Interoperability Experience. P. 1-11. 
5. Stagnaro C, Freed Associates (2017) Innovative Blockchain Uses in Health Care. Anonymous. Bitcoin Online Developer Guide, Bitcoin p. $1-13$.

6. Broder et al. (2016) Accenture, p. 5.

7. Das R (2017) Does Blockchain Have a Place in Healthcare? Pharma \& Healthcare, Forbes, USA.

8. Weiss M (2015) How Bitcoin's Technology Could Reshape Our Medical Experience, Coindesk, USA.

9. Miliard M (2017) Blockchain's potential use cases for healthcare: hype or reality? Heathcare IT news, Australia.
10. Eckblaw A, Azaria A, Hamalka J, Lippman A (2016) A Case Study for Blockchain in Healthcare: MedRec prototype for electronic health records and medical research data. MIT Media Lab. p. 1-13.

11. Bresnick J (2017) Five Blockchain Use Cases for Healthcare Payers. Health IT Analytics, USA.

12. Peters AW, Till BM, Meara JG, Afshar S (2017) Blocktrain technology in health care: A primer for surgeons. Bulletin of the American College of Surgeons, USA.

13. Linn L (2017) Blockchain for health data and its potential use in health IT and health care-related research. p. 1-10.

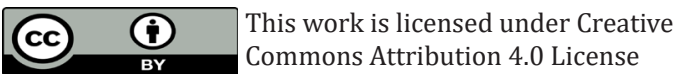

To Submit Your Article Click Here:

Submit Article

DOI: $10.32474 /$ OAJBEB.2018.01.000111

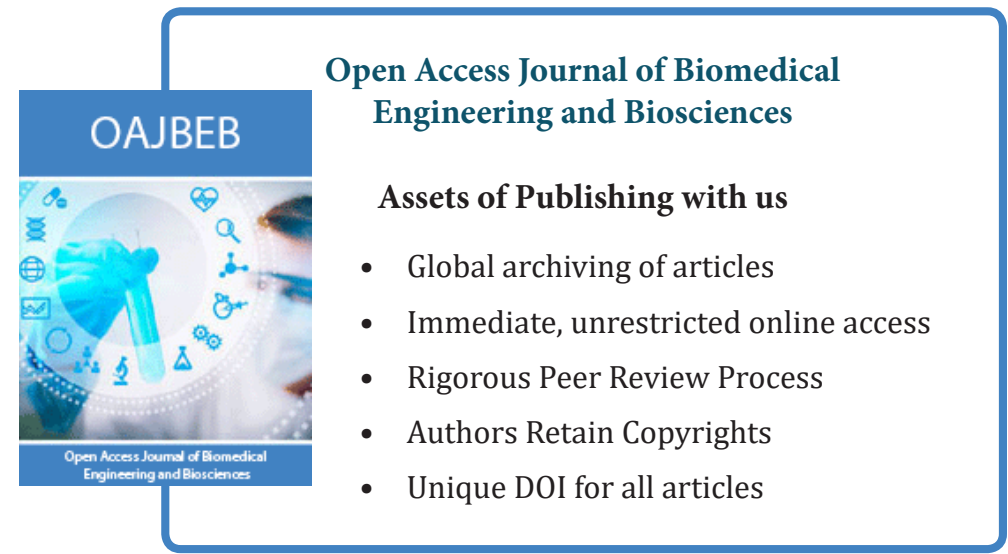

